\title{
Evaluación de los indicadores de estrés calórico en las principales localidades de lechería intensiva del departamento de Lima, Perú
}

\author{
Evaluation of heat stress indicators in the main locations of intensive dairy \\ production in Lima, Peru
}

Luis Felipe Ruiz G. ${ }^{1,4}$, Fernando Carcelén C. ${ }^{2}$, Rocío Sandoval-Monzón ${ }^{3}$

\section{Resumen}

El objetivo de este trabajo fue evaluar los indicadores de estrés calórico en las principales localidades de lechería intensiva del departamento de Lima. Se evaluaron las localidades de Végueta, Huacho, Huaral, Lurín y Cañete durante el periodo 2010-2013. Se determinó el promedio mensual y por localidad de la temperatura ambiental, la humedad relativa y el índice temperatura-humedad, la duración del estrés calórico y el índice de sobrecarga calórica (ITH Sobrecarga). Se calculó el porcentaje de días en los cuales el ITH Sobrecarga fue superior a 0 y el porcentaje de días según severidad del estrés calórico. Se demuestra que el ITH Sobrecarga fue mayor a 10 desde noviembre a mayo, con un pico en febrero. La duración del estrés calórico fue mayor a 12 horas entre enero y abril. Cañete fue la localidad con mayor ITH Sobrecarga y mayor duración de estrés calórico. Las vacas en Vegueta presentaron algún grado de estrés calórico en el 98\% de los días del año. La severidad de estrés calórico por localidad fue de Cañete $>$ Végueta $>$ Huacho $>$ Huaral $>$ Lurín. Se concluye que el ganado lechero de la zona de Lima está sometido a estrés calórico durante casi todo el año, siendo la localidad de Cañete la más afectada.

Palabras clave: índice temperatura-humedad; índice de sobrecarga calórica; estrés calórico; vacas lecheras

\footnotetext{
${ }^{1}$ Grupo de Investigación en Reproducción y Sanidad Animal, Facultad de Medicina Veterinaria, Universidad Nacional Mayor de San Marcos, Lima, Perú

${ }^{2}$ Laboratorio de Bioquímica y Nutrición Animal, Facultad de Medicina Veterinaria, Universidad Nacional Mayor de San Marcos, Lima, Perú

${ }^{3}$ Clínica de Animales Mayores, Facultad de Medicina Veterinaria, Universidad Nacional Mayor de San Marcos, Lima, Perú

${ }^{4}$ E-mail:lfrg81@hotmail.com
}

Recibido: 28 de junio de 2018

Aceptado para publicación: 21 de noviembre de 2018 
The aim of this study was to evaluate heat stress indicators in the main locations of intensive dairy production of the department of Lima. The localities of Végueta, Huacho, Huaral, Lurín and Cañete were evaluated during the period 2010-2013. The monthly and local average of air temperature, relative humidity and temperature-humidity index, the duration of the heat stress and the heat overload index (ITH Overload) were calculated. The percentage of days in which ITH Overload was greater than 0 and the percentage of days according to severity of heat stress were calculated. It was showed that the ITH Overload was greater than 10 from November to May, with a peak in February. The duration of the heat stress was greater than 12 hours between January and April. Cañete was the location with the highest ITH Overload and longest duration of heat stress. The cows in Vegueta suffered some degree of heat stress in $98 \%$ of the days of the year. The severity of heat stress by location was Cañete $>$ Végueta $>$ Huacho $>$ Huaral $>$ Lurín. It is concluded that dairy cattle in the Lima area are subjected to heat stress during most of the year, being the area of Cañete the most affected.

Key words: temperature-humidity index; heat overload index; heat stress; dairy cows

\section{INTRODUCCIÓN}

Animales expuestos a ambientes calurosos no pueden disipar el excedente del calor producido por su metabolismo y del calor del medio ambiente, por lo que se genera hipertermia (Aréchiga-Flores y Hansen, 2003; West, 2003). Cuando esta situación persiste, entonces se está ante una situación de estrés calórico (Mader et al., 2006; Martínez, 2006). El ganado vacuno elimina calor mediante mecanismos no evaporativos y evaporativos. Los mecanismos no evaporativos (conducción y convección) son poco eficientes cuando se incrementa la temperatura ambiental, quedado el ganado más dependiente del enfriamiento evaporativo (sudoración y jadeo). Sin embargo, los mecanismos evaporativos también fallan en ambientes de elevada humedad relativa. Es por eso que ambientes cálidos y húmedos no permiten que el animal pueda disipar suficiente calor corporal para prevenir el estrés calórico (West, 2003).

El estrés calórico ha sido ampliamente reconocido como un factor que afecta la productividad, la eficiencia reproductiva y la sa- lud de los animales de producción (Allen et al., 2013; Boni et al., 2014; Dash et al., 2016; Ruiz-García et al., 2018). Se puede observar una leve disminución en la producción en el ganado lechero en periodos cortos de estrés calórico, pero las consecuencias pueden ser graves en periodos prolongados de estrés calórico (St-Pierrre et al., 2003; Arias y Mader, 2010; Ruiz-García et al., 2018). El ganado vacuno responde a este tipo de estrés reduciendo el consumo de alimento e incrementando el consumo de agua, en pérdida de agua por evaporación y con aumento de la frecuencia respiratoria y la temperatura corporal (West et al., 2003; Wheelock et al., 2010; Allen et al., 2015).

Se han realizado diversos estudios para identificar los indicadores de estrés calórico en el ganado vacuno (Bouraoui et al., 2002; Ravagnolo et al., 2000; West et al., 2003; Vitali et al., 2009), siendo el índice temperatura-humedad (ITH) el más empleado, pues representa adecuadamente el efecto combinado de la temperatura y humedad relativa del medio ambiente (Thom, 1959). Se le ha utilizado como indicador de estrés calórico en el hombre y en los animales (St-Pierre et 
al., 2003; West, 2003). Así mismo, se ha determinado el ITH umbral, nivel a partir del cual el ganado vacuno manifiesta estrés calórico, siendo de 70 para ganado vacuno lechero adulto y de 72 y 77 para terneras y vaquillas lecheras, respectivamente (St-Pierre et al., 2003).

El ITH a lo largo del día se puede representar mediante una función senoidal. En base a esto, St-Pierre et al. (2003) propusieron nuevos índices para estimar el estrés calórico. Entre estos, la duración del estrés calórico (D) y el índice de sobrecarga calórica (ITH Sobrecarga). La duración del estrés calórico es la cantidad de tiempo que el ITH del ambiente excede el ITH umbral, mientras que el ITH Sobrecarga es la integral de la curva senoidal de ITH del ambiente sobre el ITH umbral. Este último representa el calor acumulado por encima del umbral durante las 24 horas del día (St-Pierre et al., 2003; Ruiz et al., 2017). Estos indicadores permiten predecir los efectos adversos del estrés calórico sobre la producción y reproducción del ganado (St-Pierre et al., 2003).

Existen pocos estudios que han podido cuantificar el efecto del estrés calórico en el ganado lechero del Perú (Ruiz-García et al., 2018). Estos estudios se han basado principalmente en el efecto de las estaciones climáticas o en el efecto del fenómeno de El Niño sobre la producción y reproducción del ganado (Rodríguez et al., 2005; Domínguez, 2008; Contreras, 2009), encontrándose que partos en verano resultan en una menor producción de leche y una menor eficiencia reproductiva.

Estudios evidencian que las cuencas lecheras del norte y centro del Perú presentan condiciones climatológicas propicias para producir estrés calórico en el ganado durante los meses de verano (Rodríguez et al., 2005; Domínguez, 2008; Contreras, 2009; Ruiz et al., 2017). Sin embargo, la intensidad y la duración del estrés calórico no han sido medidas. El departamento de Lima posee importantes localidades de producción lechera como Lurín, Cañete, Huacho, Huaral y Végueta (INEI, 2012). Por lo tanto, el objetivo de este trabajo fue determinar la severidad y duración del estrés calórico en el ganado bovino de las cinco principales localidades de lechería intensiva del departamento de Lima mediante los indicadores del estrés calórico.

\section{Materiales y Métodos}

El estudio se realizó en cinco localidades de importancia para la ganadería lechera intensiva del departamento de Lima (INEI, 2012): Végueta, Huacho, Huaral, Lima y Cañete. Se recolectaron 6512 registros diarios de temperatura máxima, temperatura mínima, humedad relativa máxima, y humedad relativa mínima, obtenidos por el Servicio Nacional de Meteorología e Hidrografía (SENAMHI) desde enero de 2010 a agosto de 2013 de las estaciones meteorológicas de dichas localidades. En el departamento de Lima, el verano ocurre entre los meses de diciembre a marzo y el invierno entre los meses de junio a setiembre.

Se determinó el promedio de la temperatura ambiental máxima ( $\mathrm{T}^{\circ}$ Máx.), la temperatura ambiental mínima ( $\mathrm{T}^{\circ}$ Mín.), la humedad relativa máxima (HR Máx.), la humedad relativa mínima (HR Mín.) por cada mes y localidad. También se determinó el índice temperatura-humedad mínimo (ITH Mín.), el índice temperatura-humedad máximo (ITH Máx.), el tiempo (D) que el ganado estuvo sometido a estrés calórico y el índice de sobrecarga calórica (ITH Sobrecarga) por mes y localidad.

Además, se calculó el porcentaje de días donde los valores de ITH Sobrecarga fueron mayores a cero para cada localidad, así como el porcentaje de días en que los valores de ITH Máx. se encontraron en las siguientes categorías: a) sin estrés calórico: ITH Máx. menor que $70, b)$ estrés calórico moderado: ITH Máx. entre 70 y 77, c) estrés calórico 
severo: ITH Máx. entre 77 y 88, y d) estrés calórico muy severo: ITH Máx. mayor que 88.

Se asumió que la temperatura y la humedad son variables anticíclicas durante el transcurso del día (Ravagnolo et al., 2000; St-Pierre et al., 2003). También se asumió que el ITH sigue una función senoidal perfecta (St-Pierre et al., 2003). Por ello, el ITH Máx. y el ITH Mín. se calcularon empleando una ecuación estándar para el cálculo del ITH (Ravagnolo et al., 2000; Bouraoui et al., 2002). El ITH Mín. se calculó utilizando la T Mín. y la HR Máx., mientras que el ITH Máx. se calculó utilizando la T Máx. y la HR Mín. Los indicadores de estrés calórico fueron calculados según las ecuaciones descritas por St-Pierre et al. (2003). Se consideró el ITH umbral de 70 como el ITH umbral del ganado bovino (St. Pierre et al., 2003).

Los datos fueron analizados mediante el paquete estadístico SPSS v. 22.0 para Windows. Los datos de cada localidad y mes se resumieron a través de los años para obtener los valores medios. Estos valores fueron sometidos a la prueba de Friedman. En los casos de significancia se utilizó la prueba de comparación por pares para determinar los grupos que presentaron diferencia estadística significativa. Asimismo, se evaluó el porcentaje de días con valores de ITH Sobrecarga mayor que cero en cada localidad empleando un modelo de regresión logística binaria y se evaluó el porcentaje de días según severidad del estrés calórico empleando un modelo de regresión ordinal. En ambos modelos, la variable independiente fue la localidad. En caso de la variable independiente resultara significativa, se estimaron los coeficientes de regresión para cada una de las combinaciones posibles y se realizó una comparación múltiple para determinar entre que grupos existía diferencia estadística significativa. En todos los análisis se empleó un nivel de significancia de $\mathrm{p}<0.05$.

\section{Resultados y Discusión}

Las variables medioambientales se presentan en la Figura 1. Se observa que la HR Máx. no varió notoriamente a lo largo del año; sin embargo, la HR Mín., la $\mathrm{T}^{\circ}$ Máx. y la $\mathrm{T}^{\circ}$ Mín. muestran una variación de distribución cíclica durante el año.

Los promedios más altos de HR Máx. y HR Mín. se presentaron en julio (HR Máx.=95.7\% y HR Mín.=79.7\%) y agosto (HR Máx.=95.9\% y HR Mín.=79.2\%), donde la HR Mín. en julio fue significativamente mayor que en los demás meses $(\mathrm{p}<0.05)$ y la HR Mín. en febrero $(68.1 \%)$ fue significativamente menor $(\mathrm{p}<0.05)$. Por otro lado, los promedios de HR Máx. en los meses de verano fueron similares, y superior al 95\% en todos los meses del año.

La $\mathrm{T}^{\circ}$ Máx. y la $\mathrm{T}^{\circ}$ Mín. variaron de forma similar forma durante el año. Los meses de verano y de invierno presentaron la mayor y menor temperatura ambiental, respectivamente. Febrero presentó los mayores promedios de $\mathrm{T}^{\circ}$ Máx. $\left(27.8^{\circ} \mathrm{C}\right)$ y $\mathrm{T}^{\circ}$ Mín. $\left(19.0^{\circ} \mathrm{C}\right)$, mientras que agosto presentó los menores promedios $\left(\mathrm{T}^{\circ}\right.$ Máx.: $20.1^{\circ} \mathrm{C}$ y $\mathrm{T}^{\circ}$ Mín.: $13.8^{\circ} \mathrm{C}$ ). Los promedios de $\mathrm{T}^{\circ}$ Máx. $\mathrm{y}$ $\mathrm{T}^{\circ}$ Mín. en los meses de verano fue superior a los 25 y $18{ }^{\circ} \mathrm{C}$, respectivamente.

Los promedios de $\mathrm{T}^{\circ}$ Máx., $\mathrm{T}^{\circ}$ Mín., $\mathrm{HR}$ Máx. y HR Mín. por localidad se muestran en el Cuadro 1. Cañete presentó el mayor promedio de $\mathrm{T}^{\circ}$ Máx. $\left(27.4{ }^{\circ} \mathrm{C}\right)$ en comparación con otras localidades $(\mathrm{p}<0.05)$, en tanto que Huaral presentó la menor $\mathrm{T}^{\circ}$ Máx. $\left(22.4^{\circ} \mathrm{C}\right)$, siendo significativamente inferior a las demás localidades $(\mathrm{p}<0.05)$. Por otro lado, la $\mathrm{T}^{\circ}$ Mín. de Huacho $\left(16.5^{\circ} \mathrm{C}\right)$ y Lurín $\left(16.7^{\circ} \mathrm{C}\right)$ fueron significativamente superiores a la $\mathrm{T}^{\circ}$ Mín. de Cañete $\left(15.7^{\circ} \mathrm{C}, \mathrm{p}<0.05\right)$. 


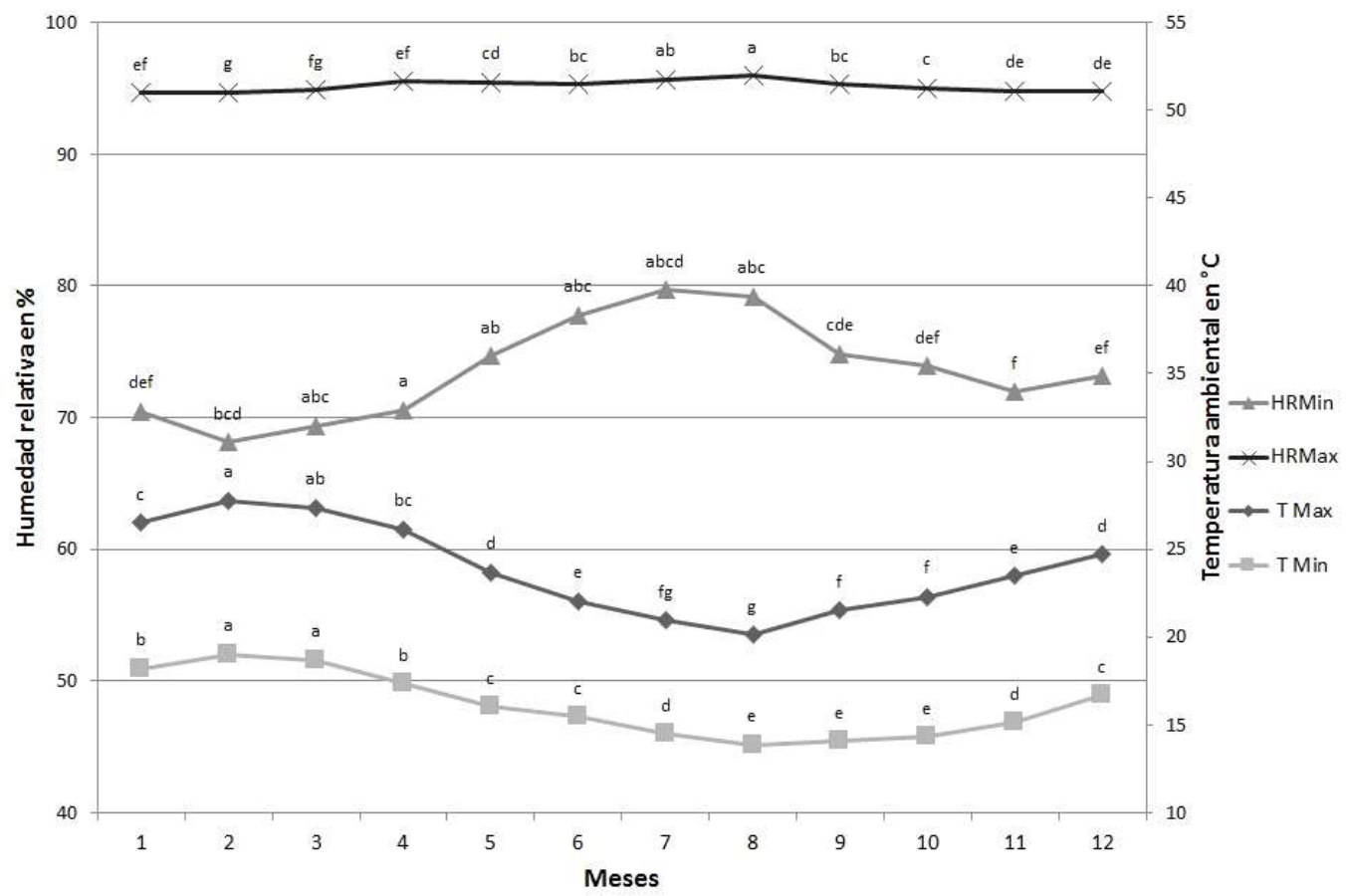

Figura 1. Valores promedios de la temperatura máxima ( $\mathrm{T}^{\circ}$ Máx. ) y mínima $\left(\mathrm{T}^{\circ}\right.$ Mín. $)$ en grados centígrados, y la humedad relativa máxima (HR Máx.) y mínima (HR Mín.) en porcentaje, para los meses del año en el departamento de Lima entre el periodo 2010 y 2013. Letras diferentes indican diferencia estadística significativa $(\mathrm{p}<0.05)$ dentro de cada variable

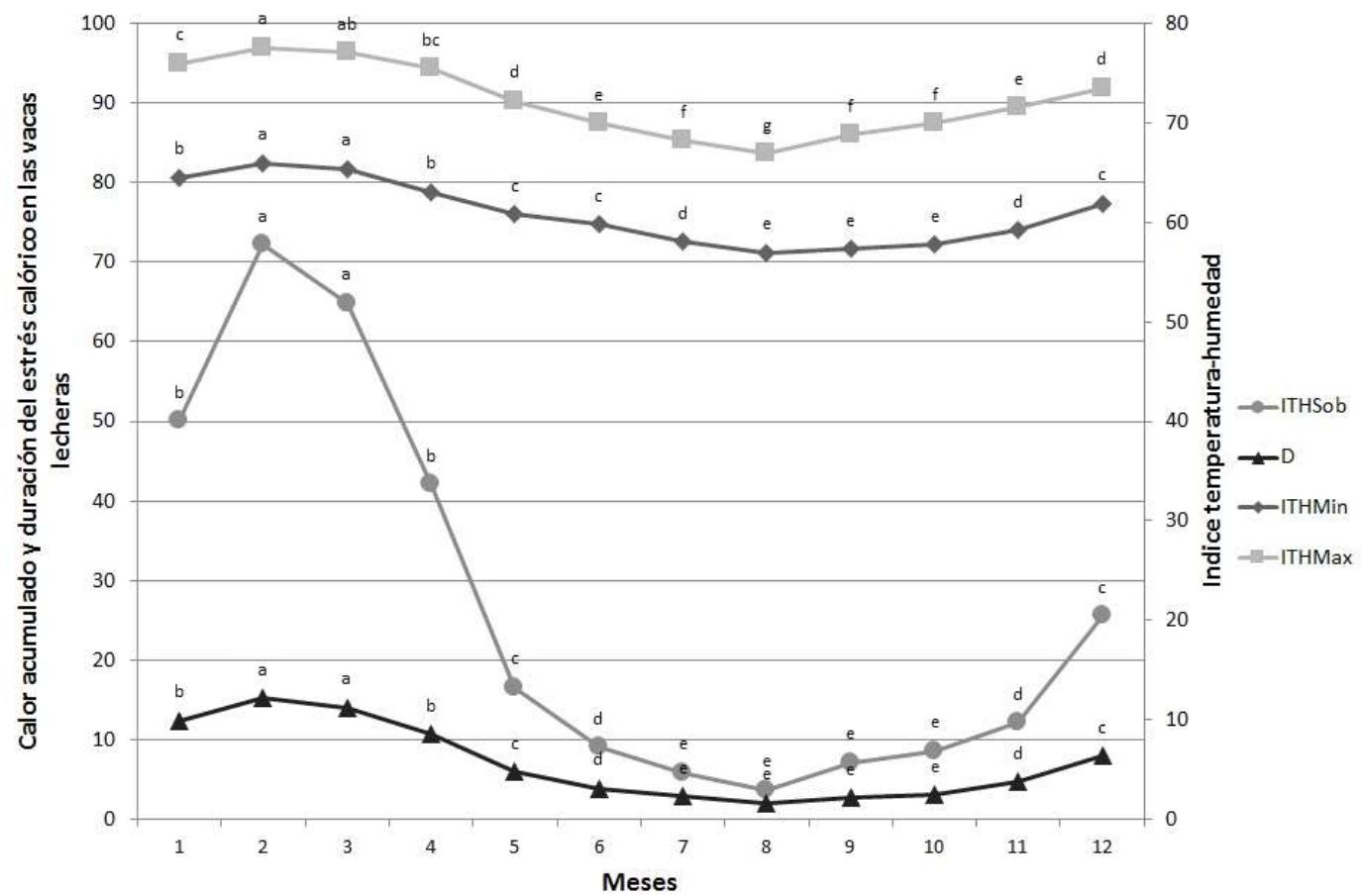

Figura 2. Valores promedios del indice de temperatura-humedad maximo (II H Máx.) y minımo (ITH Mín.), la sobrecarga de calor (ITH Sobrecarga) y la duración (D) del estrés calórico por mes en el departamento de Lima (2010-2013). Letras diferentes indican diferencia estadística significativa $(\mathrm{p}<0.05)$ dentro de cada variable 


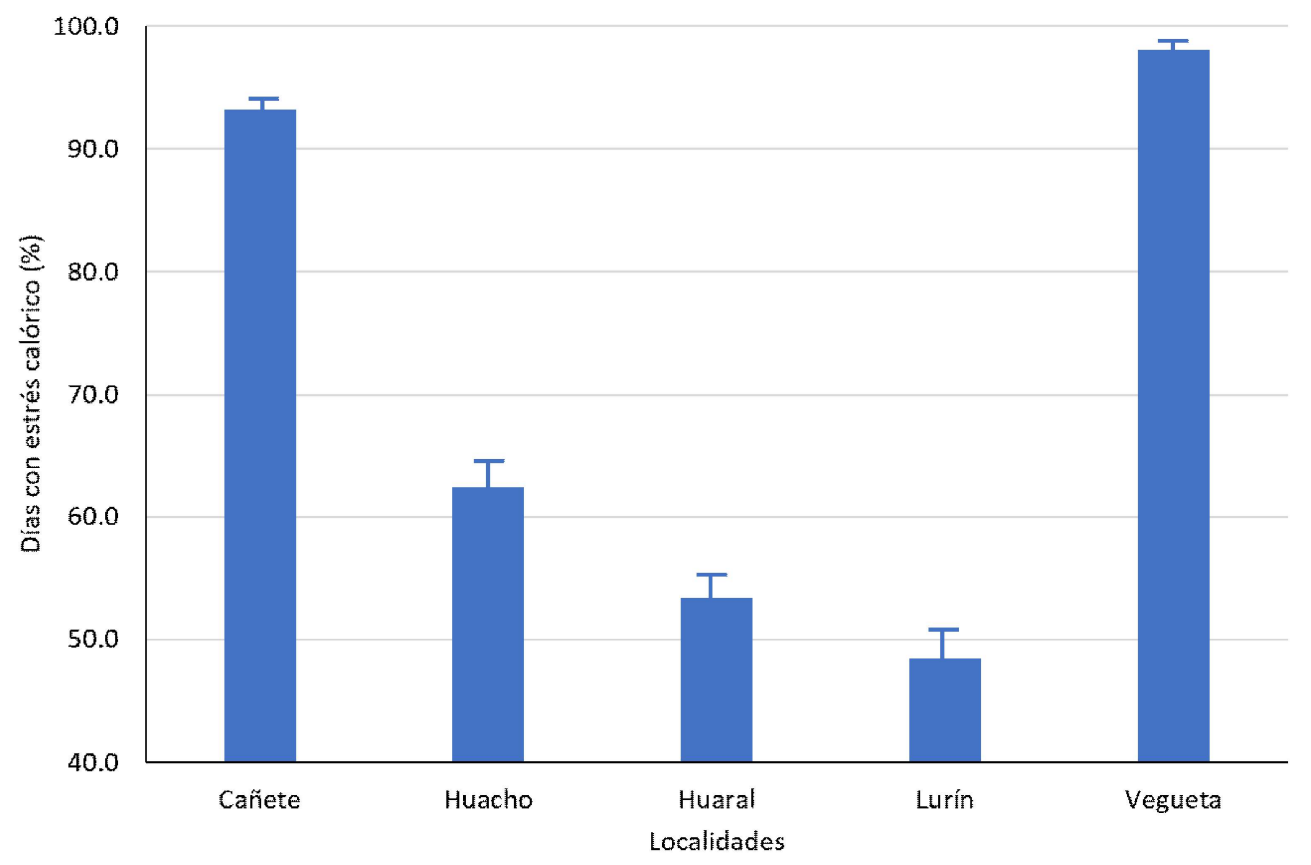

Figura 3. Porcentajes y límites de confianza al $95 \%$ de días con sobrecarga de calor superior a cero en cinco localidades del departamento de Lima (2010-2013). Letras diferentes indican diferencia estadística significativa $(\mathrm{p}<0.05)$

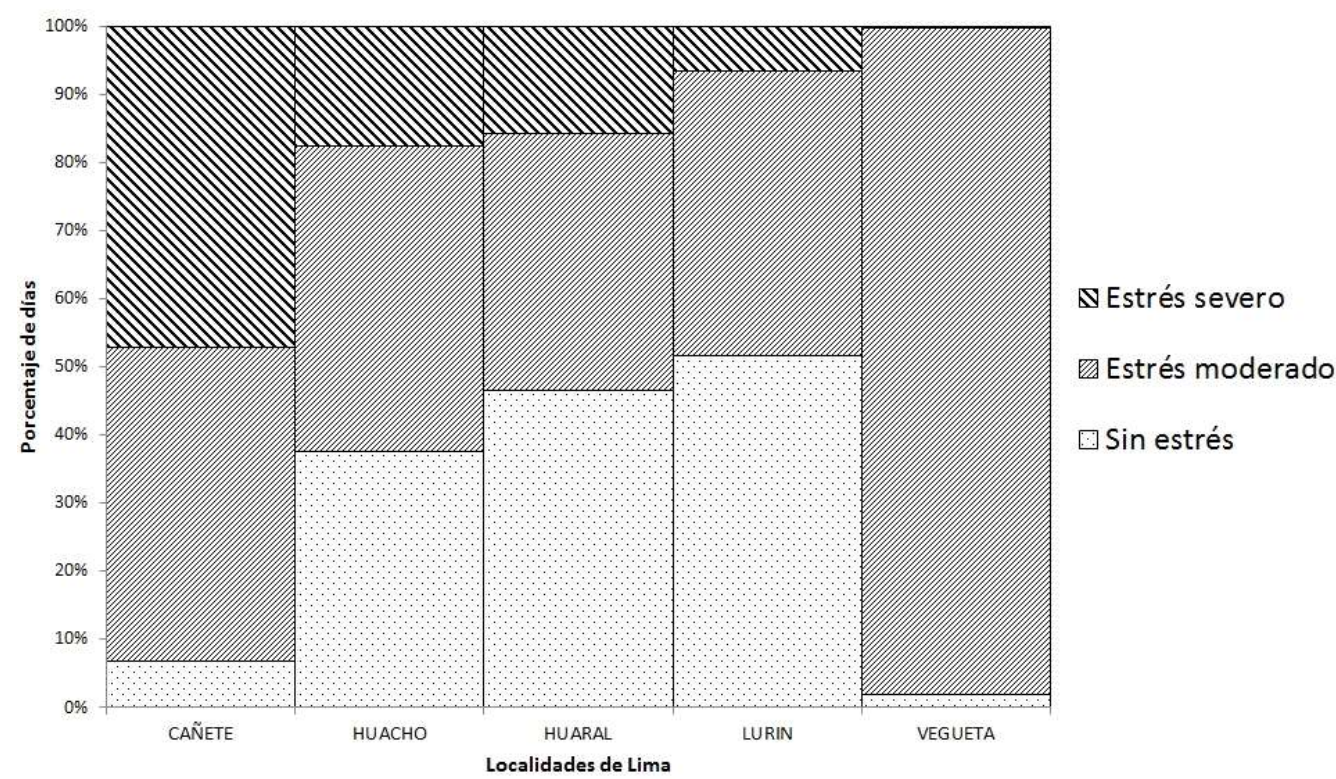

Figura 4. Gráfico mosaico por nivel de estrés calórico para las vacas lecheras de cinco localidades del departamento de Lima (periodo 2010-2013). Sin estrés calórico $=$ ITH Máx. $<70$; estrés calórico moderado $=$ ITH Máx. $>70 \mathrm{y}<77$; estrés calórico severo $=$ ITH Máx. $>77$ 
Cuadro 1. Valores promedios y límites de confianza (LC) al 95\% de temperatura máxima ( $\mathrm{T}^{\circ}$ Máx.) y mínima ( $\mathrm{T}^{\circ}$ Mín.) y humedad relativa máxima (HR Máx.) y mínima (HR Mín.) en cinco localidades del departamento de Lima (2010-2013)

\begin{tabular}{lcccccccc}
\hline & \multicolumn{2}{c}{$\mathrm{T}^{\circ}$ Máx. $\left({ }^{\circ} \mathrm{C}\right)$} & \multicolumn{2}{c}{$\mathrm{T}^{\circ}$ Mín. $\left({ }^{\circ} \mathrm{C}\right)$} & \multicolumn{2}{c}{ HR Máx. $(\%)$} & \multicolumn{2}{c}{ HR Mín. $(\%)$} \\
\cline { 2 - 9 } & Media & $\pm \mathrm{LC}$ & Media & $\pm \mathrm{LC}$ & Media & $\pm \mathrm{LC}$ & Media & $\pm \mathrm{LC}$ \\
\hline Cañete & $27.35^{\mathrm{a}}$ & 0.16 & $15.68^{\mathrm{d}}$ & 0.16 & $95.71^{\mathrm{c}}$ & 0.06 & $61.79^{\mathrm{e}}$ & 0.43 \\
Huacho & $23.79^{\mathrm{b}}$ & 0.20 & $16.49^{\mathrm{a}}$ & 0.14 & $92.03^{\mathrm{e}}$ & 0.18 & $70.09^{\mathrm{c}}$ & 0.55 \\
Huaral & $22.39^{\mathrm{e}}$ & 0.20 & $16.12^{\mathrm{c}}$ & 0.12 & $96.66^{\mathrm{b}}$ & 0.08 & $80.87^{\mathrm{b}}$ & 0.31 \\
Lurín & $22.77^{\mathrm{d}}$ & 0.24 & $16.72^{\mathrm{a}}$ & 0.14 & $93.48^{\mathrm{d}}$ & 0.22 & $65.74^{\mathrm{d}}$ & 0.69 \\
Végueta & $23.67^{\mathrm{c}}$ & 0.06 & $16.18^{\mathrm{b}}$ & 0.04 & $97.75^{\mathrm{a}}$ & 0.02 & $88.61^{\mathrm{a}}$ & 0.16 \\
\hline
\end{tabular}

$a, b, c, d$ Letras diferentes dentro de columnas indican diferencia significativa $(p<0.05)$

Cuadro 2. Valores promedios y límites de confianza (LC) al 95\% del índice de temperaturahumedad máximo (ITH Máx.) y mínimo (ITH Mín.), la sobrecarga de calor (ITH Sobrecarga) y la duración (D) del estrés calórico en vacas lecheras de cinco localidades del departamento de Lima (2010-2013)

\begin{tabular}{lcccccccc}
\hline & \multicolumn{2}{c}{ ITH Máx. } & \multicolumn{2}{c}{ ITH Mín. } & \multicolumn{2}{c}{ ITH Sobrecarga } & \multicolumn{2}{c}{ D } \\
\cline { 2 - 9 } & Media & \pm LC & Media & \pm LC & Media & \pm LC & Media & \pm LC \\
\hline Cañete & $76.25^{\mathrm{a}}$ & 0.22 & $60.19^{\mathrm{c}}$ & 0.29 & $51.60^{\mathrm{a}}$ & 2.21 & $10.26^{\mathrm{a}}$ & 0.27 \\
Huacho & $71.77^{\mathrm{b}}$ & 0.27 & $61.49^{\mathrm{a}}$ & 0.24 & $26.34^{\mathrm{c}}$ & 1.84 & $6.92^{\mathrm{b}}$ & 0.35 \\
Huaral & $70.64^{\mathrm{c}}$ & 0.29 & $60.96^{\mathrm{c}}$ & 0.22 & $21.40^{\mathrm{d}}$ & 1.65 & $5.70^{\mathrm{c}}$ & 0.33 \\
Lurín & $69.74^{\mathrm{d}}$ & 0.29 & $61.92^{\mathrm{a}}$ & 0.25 & $18.81^{\mathrm{e}}$ & 1.55 & $5.91^{\mathrm{c}}$ & 0.41 \\
Végueta & $73.56^{\mathrm{b}}$ & 0.08 & $61.08^{\mathrm{b}}$ & 0.08 & $21.36^{\mathrm{b}}$ & 0.61 & $8.35^{\mathrm{b}}$ & 0.12 \\
\hline
\end{tabular}

$a, b, c, d$ Letras diferentes dentro de columnas indican diferencia significativa $(p<0.05)$

Con respecto a la HR Máx., las localidades de Végueta y Huacho presentaron el mayor $(97.8 \%)$ y menor promedio $(92.0 \%)$, respectivamente, siendo ambas significativamente diferentes a las otras localidades $(\mathrm{p}<0.05)$. Del mismo modo, Végueta presentó el mayor promedio de HR Mín. (88.6\%) y Cañete la menor (61.8\%), siendo ambas significativamente diferentes a las otras localidades $(\mathrm{p}<0.05)$.
La Figura 2 muestra que tanto el ITH Máx. como el ITH Mín. siguen un patrón de distribución similar durante el año, observándose valores más altos durante el verano. El pico del ITH Máx. y del ITH Mín. se presentaron en febrero (ITH Máx.=78, ITH Mín.=66). Los valores mínimos del ITH Máx. y del ITH Mín. se presentaron en invierno. Los valores más bajos del ITH Máx. y del ITH Mín. se observaron en agosto (ITH 
Máx.=67, ITH Mín.=57). Los valores del ITH Máx. y del ITH Mín. en febrero y agosto fueron significativamente diferentes a los demás meses $(\mathrm{p}<0.05)$. El promedio mensual del ITH Máx. superó el 70\% entre octubre y junio.

También se puede observar que el ITH Sobrecarga fue mayor a cero todo el año, considerando un ITH umbral de 70. El ITH Sobrecarga fue mayor a 10 desde de noviembre hasta mayo, con un pico en febrero (ITH Sobrecarga $=72$ ). El estrés calórico fue mayor durante los meses de verano, siendo superior a 12 horas en enero, febrero, marzo y abril. La mayor duración del estrés calórico ocurrió en febrero $(15 \mathrm{~h}, \mathrm{p}<0.05)$.

La localidad de Cañete presentó el ITH Máx. promedio más alto (ITH Máx. $=76.3$ ) seguido por Végueta (ITH Máx. =73.6), siendo estadísticamente diferentes entre ellas y entre las otras localidades $(\mathrm{p}<0.05)$. Lurín fue la localidad que tuvo el ITH Máx. más bajo (ITH Máx. =69.7), pero presentó el ITH Mín. más alto (ITH Mín. = 61.9), aunque sin diferencias significativas a otras localidades (Cuadro 2).

Cañete fue la localidad que presentó el mayor ITH sobrecarga (ITH Sobrecarga $=$ $51, \mathrm{p}<0.05$ ). Así mismo, la duración del estrés calórico en esta localidad (10 horas/día) fue significativamente superior a las demás localidades $(\mathrm{p}<0.05)$. La segunda localidad con mayor duración de estrés calórico fue Végueta ( 8 horas/día).

El porcentaje de días que el ITH Sobrecarga superó el cero en cada localidad durante los tres años del estudio se muestra en la Figura 3. Las localidades de Végueta y Cañete presentan casi la totalidad de días con un ITH Sobrecarga mayor a cero, mientras que Huacho, Huaral y Lurín presentan cerca de la mitad de los días con algún grado de estrés calórico. Se encontró que Végueta exhibe algún grado de estrés calórico en el 98\% de los días del año, seguido por Cañete con e1 $93 \%$ de los días. Luego se encuentran las localidades de Huacho (62\%), Huaral $(53 \%)$ y Lurín $(48 \%)(\mathrm{p}<0.05)$.

En la Figura 4 se observa el porcentaje de días que los valores de ITH Máx. se encontraron en alguna de las cuatro categorías de estrés calórico en las cinco localidades de Lima. La localidad de Cañete presentó el 93\% de días con un ITH Máx. superior a 70, de los cuales el $46 \%$ presentó un ITH Máx. entre 70 y 77 (estrés calórico moderado) y el $47 \%$ entre 77 y 88 (estrés calórico severo). Por otro lado, la localidad de Végueta presentó el 98\% de días con un ITH Máx. superior a 70; sin embargo, la casi totalidad de días presentó un estrés calórico moderado. Así mismo, si bien las localidades de Huacho, Huaral y Lurín presentaron menos días de estrés calórico, algunos días fueron bajo estrés calórico severo. Según los resultados del análisis estadístico, la severidad de estrés calórico por localidad fue de la siguiente manera: Cañete $>$ Végueta $>$ Huacho $>$ Huaral $>$ Lurín.

Los resultados del estudio comprueban que el estrés calórico es un problema en las vacas lecheras de las principales localidades de lechería intensiva del departamento de Lima, tal y como había sido demostrado previamente para las terneras y las vaquillas lecheras de la zona (Ruiz et al., 2017). Los resultados indican que el estrés calórico no solo ocurre en las vacas lecheras durante los meses de verano, como es creído por la mayoría de los ganaderos y profesionales vinculados a los temas ganaderos, sino que es un problema que está presente en la mayoría de los meses del año (Flamenbaum, 2011). Esto se debe principalmente a que la interacción de la T Máx. promedio diaria (entre 20 y $29^{\circ} \mathrm{C}$ ) y la HR Mín. promedio diaria (entre 69 y $80 \%$ ) producen un ITH Máx. que va entre 67 a 78 para cada mes. El valor más bajo del ITH Máx. se encuentra muy cerca del ITH umbral para el ganado vacuno (ITH umbral=70) (St-Pierre et al., 2003). 
El ITH Sobrecarga para las vacas lecheras fue mayor a cero en todos los meses del año a diferencia de lo que ocurre en las terneras y vaquillas lecheras (Ruiz et al., 2017). Era de esperarse que la sobrecarga de calor acumulada por día en los meses de verano sea mucho mayor que en los meses de invierno, pero lo inesperado fue encontrar que las vacas también registran sobrecarga de calórica en los meses de invierno.

El ganado vacuno lechero de Lima procede de países de baja humedad relativa y temperatura ambiental (West, 2003), que manifiesta estrés calórico cuando el ITH umbral llega a 70 . Aunque es posible que exista una adaptación de las vacas lecheras al medio ambiente de Lima y que los efectos adversos del estrés calórico no sean muy notorios, no se dispone de muchos estudios que muestren la magnitud del estrés calórico en el país (Rodríguez et al., 2005; Domínguez, 2008; Contreras, 2009; Flamenbaum, 2011). En este sentido, Ruiz-García et al. (2018) encontraron que la producción de leche diaria disminuye en $0.36 \mathrm{~kg}$ con el incremento de una unidad del ITH máximo. Por otro lado, el uso de la inseminación artificial con semen importado de regiones más frías y menos húmedas no favorecería el proceso de adaptación por selección, por lo que es muy probable que las hijas de estos toros no puedan expresar todo su potencial en el ambiente de Lima como ha sido demostrado en otros países (Ravagnolo et al., 2000; Bouraoui et al., 2002; West et al., 2003).

La duración del estrés calórico en los meses del verano también es otro punto preocupante. La mayoría de los ganaderos de la región consideran que las vacas solo necesitan ventilación y baños por aspersión al mediodía o cuando van a ser ordeñadas. Sin embargo, como demuestran los resultados del estudio, el estrés calórico tiene una duración que supera las 10 horas diarias en los meses de verano. Esto significa que las vacas necesitarían de procedimientos de ventilación y enfriamiento más frecuentes y por periodos más prolongados para poder disminuir los efectos adversos del estrés calórico (StPierre et al., 2003; Flamenbaum, 2011).

El estrés calórico en las localidades de Cañete y Végueta tienen un estrés calórico más intenso que el presentado en las regiones de Chillán, Curicó, Osorno y Temuco en Chile (Arias y Mader, 2010). En la localidad de Cañete las vacas lecheras sufren estrés calórico 10 horas diarias en promedio al año y una sobrecarga de calórica diaria de 51 en promedio al año, similar a lo que ocurre en las Florida y Texas, EEUU, donde las vacas pasan 11.7 y 8.7 horas diarias en estrés calórico y con una sobrecarga de calor diaria de 77 y 70 unidades, respectivamente (St-Pierre et al., 2003). En la localidad de Végueta, las vacas pasaron 8 horas diarias en estrés calórico y tuvieron una sobrecarga promedio de 21 , lo cual es comparable con lo que sucede en el estado de Arizona, EEUU, donde las vacas pasan 5.2 horas diarias en estrés calórico y con una sobrecarga de calor diaria de 33 unidades (St-Pierre et al., 2003). Sin embargo, a diferencia de lo que ocurren en dichas zonas, los establos lecheros de Lima no cuentan con sistemas adecuados para el control de los efectos del estrés calórico (Flamenbaum, 2011).

\section{Conclusiones}

- El estrés calórico de las vacas lecheras en el departamento de Lima no está delimitado a los meses de verano (diciembremarzo), sino que sufren de diversos grados de estrés calórico durante todo el año.

- Las vacas de las localidades de Cañete y Végueta presentan el mayor porcentaje de días y mayor severidad de estrés calórico que las vacas de las localidades de Huacho, Huaral y Lurín.

- La duración del estrés calórico supera las 10 horas diarias en los meses de verano. 


\section{Agradecimientos}

Los autores del estudio agradecen los datos proporcionados por el Servicio Nacional de Meteorología e Hidrología de Perú (SENAMHI-Perú), sin los cuales no se habría poder realizado el estudio.

\section{Literatura Citada}

1. Allen JD, Hall LW, Collier RJ, Smith JF. 2015. Effect of core body temperature, time of day, and climate conditions on behavioral patterns of lactating dairy cows experiencing mild to moderate heat stress. J Dairy Sci 98: 118-127. doi: 10.3168/jds.2013-7704

2. Aréchiga-Flores C, Hansen P. 2003. Efectos climáticos adversos en la función reproductiva de los bovinos. Vet Zacatecas 2: 89-107.

3. Arias R, Mader T. 2010. Determination of potential risk of heat stress of cattle in four locations of central and southern Chile. Arch Med Vet 42: 33-39. doi: 10.4067/S0301-732X2010000100005

4. Boni R, Perrone LL, Cecchini S. 2014. Heat stress affects reproductive performance of high producing dairy cows bred in an area of southern Apennines. Livest Sci 160: 172-177. doi: 10.1016/ j.livsci.2013.11.016

5. Bouraoui R, Lahmar M, Majdoub A, Djemali M, Belyea R. 2002. The relationship of temperature-humidity index with milk production of dairy cows in a Mediterranean climate. Anim Res 51: 479-491. doi: 10.1051/animres:-2002036

6. Contreras M. 2009. Efecto del estrés calórico sobre la producción en un establo lechero de la zona de Trujillo. Tesis de Ingeniero Zootecnista. Lima, Perú: Univ. Nacional Agraria La Molina. 26 p.

7. Dash S, Chakravarty AK, Singh A, Upadhyay A, Singh M, Yousuf S. 2016. Effect of heat stress on reproduc-tive performances of dairy cattle and buffaloes: a review. Vet World 9: 235-244. doi: 10.14202/vetworld.-2016.235-244
8. Domínguez C. 2008. Efectos del estrés calórico en vacas lecheras de Lima. Tesis de Ingeniero Zootecnista. Lima, Perú: Univ. Nacional Agraria La Molina. 37 p.

9. Flamenbaum I. 2011. Relación costobeneficio del sistema de enfriamiento de vacas lecheras en el verano en la zona costera de Perú. Mundo Ganadero 2:10-12.

10. [INEI] Instituto Nacional de Estadística e Informática. 2012. IV Censo nacional agropecuario 2012. [Internet]. Disponible en: http://desa.inei.gob.pe/ cenagro/redatam/?id=censosnacionales\#

11. Mader T, Davis M, Brown-Brandl T. 2006. Environmental factors influencing heat stress in feedlot cattle. J Anim Sci 84: 712-719. doi: $10.2527 / 2006.843712 x$

12. Martínez, A. 2006. Efectos climáticos sobre la producción del vacuno lechero: estrés de calor. REDVET 7(10). [Internet]. Disponible en: http:// www.produccion-animal.com.ar/clima y_ambientacion/30-stres_por_ calor_vaca_lechera.pdf

13. Ravagnolo O, Misztal I, Hoogenboom G. 2000. Genetic component of heat stress in dairy cattle, development of heat index function. J Dairy Sci 8: 2120-2125. doi: 10.3168/jds.S0022-0302(00)75094-6

14. Rodríguez, L, Ara M, Huamán H, Echevarría L. 2005. Modelos de ajuste para curvas de lactación de vacas en crianza intensiva en la cuenca de Lima. Rev Inv Vet Peru 16: 1-12. doi: 10.15381/ rivep.v16il.1517

15. Ruiz-García LF, Carcelén-Cáceres F, Sandoval-Monzón RS. 2018. El índice temperatura-humedad máximo y la producción de leche de los establos en LimaPerú. Arch Zootec 68: 99-107. doi: 10.21071/az.v67i257.3497

16. Ruiz LF, Sandoval R, Pizarro J, Carcelén F. 2017. Severidad y duración del estrés calórico en terneras y vaquillas de las principales localidades de lechería intensiva del departamento de Lima, Perú. Rev Inv Vet Peru 28: 505513. doi: 10.15381/rivep.v28i3.13368 
17. St-Pierre NR, Cobanov B, Schnitkey G 2003. Economic losses from heat stress by us livestock industries. J Dairy Sci 86: 52-77. doi: $10.3168 /$ jds.S00220302(03)74040-5

18. Thom EC. 1959. The discomfort index. Weatherwise 12: 57-59. doi: 10.1080/ 00431672.1959.9926960

19. Vitali A, Segnalini M, Bertocchi L, Bernabucci U, Nardone A, Lacetera N. 2009. Seasonal pattern of mortality and temperature humidity index in dairy cows. J Dairy Sci 92: 3781-3790. doi: $10.3168 /$ jds.2009-2127
20. West J. 2003. Effects of heat-stress on production in dairy cattle. J Dairy Sci 86:2131-2144. doi: 10.3168/jds.S00220302(03)73803-X

21. West J, Mullinix B, Bernard J. 2003. Effects of hot, humid weather on milk temperature, dry matter intake, and milk yield of lactating dairy cows. J Dairy Sci 86: 232-242. doi: 10.3168/jds.S00220302(03)73602-9.

22. Wheelock J, Rhoads R, Vanbaale M, Sanders S, Baumgard L. 2010. Effects of heat stress on energetic metabolism in lactating holstein cows. J Dairy Sci 93: 644-655. doi: 10.3168/jds.2009-2295 